28 | 2002

Histoire de l'enseignement du français langue

étrangère ou seconde dans le bassin méditerranéen.

Volume 2

\title{
Un homme des Lumières face aux langues du sud de la Méditerranée, ou Volney et sa « méthode alfabétique »
}

Henri Besse

\section{OpenEdition}

Journals

\section{Édition électronique}

URL : https://journals.openedition.org/dhfles/2681

DOI : $10.4000 /$ dhfles.2681

ISSN : 2221-4038

Éditeur

Société Internationale pour l'Histoire du Français Langue Étrangère ou Seconde

Édition imprimée

Date de publication : 1 juin 2002

Pagination : 155-176

ISSN : 0992-7654

Référence électronique

Henri Besse, «Un homme des Lumières face aux langues du sud de la Méditerranée, ou Volney et sa « méthode alfabétique » », Documents pour l'histoire du français langue étrangère ou seconde [En ligne], 28 | 2002, mis en ligne le 31 janvier 2014, consulté le 27 mai 2021. URL : http://

journals.openedition.org/dhfles/2681; DOI : https://doi.org/10.4000/dhfles.2681

Ce document a été généré automatiquement le 27 mai 2021

(c) SIHFLES 


\title{
Un homme des Lumières face aux langues du sud de la Méditerranée, ou Volney et sa « méthode alfabétique »
}

\author{
Henri Besse
}

Constantin-François Chassebeuf (ou Chasseboeuf) ne fut vraiment connu sous le nom de Volney (contraction, dit-on, de Voltaire et de Ferney) qu'à la publication, en 1787, de son Voyage en Syrie et en Egypte pendant les années 1783, 1784 et 1785 (abrégé ci-après en Voyage ${ }^{1}$ ), suivie, en 1791 quand la Révolution eut libéré sa parole, des Ruines ou Méditations sur les révolutions des empires (ci-après Ruines), qui en est une sorte d'interprétation " philosophique». Célèbre pour ces deux ouvrages, il l'est beaucoup moins en tant qu'auteur de ce qu'il finira par appeler, à la fin de sa vie, la « méthode alfabétique». Un de ses amis, le comte P.-A. Daru disait pourtant en juin 1820 (Volney 1827²: 17), peu après sa mort, que :

L'expérience qu'il avait faite de l'utilité des langues orientales lui avait fait concevoir un vif désir d'en propager la connaissance, et, pour la propager, il avait senti la nécessité de la rendre moins difficile. C'est dans cette vue qu'il conçut le projet d'appliquer à l'étude des idiomes de l'Asie une partie des notions grammaticales que nous avons acquises sur les langues européennes. [...] M. de Volney a développé son système dans trois ouvrages ${ }^{3}$ qui prouvent que cette idée [...] n'a pas cessé de l'occuper pendant vingt-cinq ans.

2 Cette " expérience " concerne surtout l'arabe, seule langue orientale que Volney fut jamais à même de parler ; ce qui n'était pas alors fréquent, même chez les " arabistes " comme on disait alors 4 . Quant à son " projet», il revient à appliquer la "science " grammaticale de l'Europe à un Orient présupposé plus ou moins " pré-scientifique » voire «a-scientifique».

De l'ensemble des écrits de C.-F. Volney, il n'existe aucune édition réellement complète, même si plusieurs se prétendent telles ${ }^{5}$. Approuvées par l'auteur avant sa mort et éditées sous le contrôle de Daru, paraissent en 1821 les CEuvres complètes de Volney ${ }^{6}$, 
lesquelles n'incluent pas - ce qui ne saurait surprendre à cette date - ses pamphlets de 1788-1789. Cette édition fut republiée en 1826, à quelques modifications orthographiques près, sous le titre Euvres de C. F. Volney"7, édition à laquelle nous nous référerons principalement ici ${ }^{8}$. Les ouvrages concernant notre propos y sont, certes, " présentés sans indication de date et dans un ordre absurde " (Gaulmier 1945-1946 : 113), mais on peut en reconstituer la chronologie à partir de la thèse de Gaulmier ([1951] 1980) et de 1' "Esquisse d'une Bibliographie Volneyenne » qu'il y propose ${ }^{9}$ : le Voyage (1787) et les Ruines (1791); la Simplification des langues orientales en 1795 (ci-après Simplification); les Vues nouvelles sur l'enseignement des langues orientales (Vues nouvelles), Gaulmier (1980 : 547, note 3) précisant qu'elles ont été rédigées « vers 1804, et en tous cas, avant 1807, date de création d'une chaire d'arabe à Marseille "; L'alfabet européen appliqué aux langues asiatiques paru en 1819 (Alfabet européen) ; L'hébreu simplifié par la méthode alfabétique (Hébreu simplifié), sous presse à la mort de Volney au printemps 1820 ; enfin, le Discours sur l'étude philosophique des langues (Discours) qu'il lut à l'Académie française « le premier mardi de décembre » 1819.

\section{Comment Boisgirais devint Volney}

4 Le père du jeune Constantin-François se faisait appeler de Boisgirais (ou de Boisgiret, toponyme d'une de ses métairies), et son fils entra sous ce nom au collège d'Ancenis, près de Nantes, puis chez les Oratoriens d'Angers. Emancipé à dix-huit ans (avec 1100 livres de rente provenant de sa mère), il part pour Paris en 1775, où il fréquente, en compagnie de P. J. Cabanis (né comme lui en 1757), les salons de Anne-Catherine Helvétius ${ }^{10}$ et du baron d'Holbach ${ }^{11}$ Selon Bossange (I, p. VIII), Boisgirais étudie alors la médecine " pendant trois années " ainsi que le grec ancien, composant « un Mémoire sur la Chronologie d'Hérodote», qu'il eut quelque peine à faire publier ${ }^{12}$, l'Eglise s'opposant à tout ce qui semblait remettre en cause sa chronologie biblique. Il suit également, de 1780 à 1782, le cours d'arabe que donne Leroux de Hauterayes (ou des Hautesrayes) au " collège royal de France ${ }^{13}$ ", où il se lie avec " $\mathrm{M}$. Venture, interprète des langues orientales, qui aujourd'hui accompagne le général Buonaparte » (II, 85, note 1$)^{14}$.

D'où lui vient cet intérêt pour l'Orient et pour ses langues ? Bossange (I, p. IX) en donne une explication à la fois prosaïque et " romantique » : " une modique succession lui échut : il résolut d'en employer l'argent à entreprendre un long voyage. Comme tous les grands hommes, il dédaigna les routes frayées, et choisit la plus inconnue et la plus périlleuse : il projeta de parcourir l'Égypte et la Syrie. » Gaulmier (1980:37) est sans doute plus proche de la vérité quand il suppose que, « comme d'Holbach, Volney sera conduit à l'histoire par le dessein d'élucider les mystérieuses origines du christianisme. "Quoi qu'il en soit, un tel projet exigeait une préparation physique, à laquelle Bossange accorde (I, pp. IX-X) plus d'importance que Gaulmier, mais aussi une préparation intellectuelle qui ne fut pas exactement ce que ce dernier en dit (en particulier dans 1945-1946 : 114). Volney explicite pourtant fort bien, entre autres dans son Alfabet européen, quelle fut sa première approche de l'arabe :

lorsque, me préparant à voyager en Syrie, je voulus acquérir les premiers élémens de la langue arabe : j'ouvris la grammaire d'Erpénius ${ }^{15}:$ ne comprenant rien à ce genre nouveau de doctrine, j'eus recours au professeur royal alors en fonction; sa patiente complaisance écouta toutes les questions et les objections dont j'avais rédigé la liste : elles lui parurent raisonnables; mais le résultat fut 'que les usages 
étant établis, l'on ne pouvait les changer ; que le but des professeurs royaux n'était pas tant d'enseigner l'arabe parlé, que l'arabe écrit, en tant qu'il contribue à expliquer les anciens livres des Juifs ; que sans doute l'arabe vulgaire avait une grande utilité commerciale et diplomatique ; mais que [...] le meilleur parti était d'apprendre la langue dans le pays même et de la bouche des naturels (VIII, 96-97).

On aura compris que Volney commença de lui-même à apprendre l'arabe dans la grammaire d'Erpenius, " par règles » et non " par usage», or ces " règles » s'y trouvaient formulées selon une « doctrine » qui n'était pas celle à laquelle il avait été jusqu'alors accoutumé, d'où son désarroi et sa visite à Hauterayes ${ }^{16}$.

7 Ce n'est pas la métalangue latine de cette grammaire ${ }^{17}$ qui embarrassa l'ancien élève des Oratoriens, mais bien, si l'on en croit toujours l'Alfabet européen, le système d'écriture propre à l'arabe et le métalangage qui informe traditionnellement celui-ci :

Il faut l'avouer, le premier aspect des alfabets orientaux frappe le disciple européen d'une sensation pénible et décourageante : la figure des lettres est étrange pour lui ; son amour-propre se sent blessé de n'y rien comprendre : déjà loin de l'enfance, il va redevenir écolier [...] ce ne sont là que des préliminaires : l'explication commence ; il a coutume d'écrire de gauche à droite, on lui ordonne d'écrire de droite à gauche ; son écriture européenne trace tout ce qui se prononce ; l'écriture asiatique, en général, n'en trace qu'une partie; il faut deviner le reste ; les professeurs royaux, étrangers à ces langues, décrivent plutôt qu'ils ne font entendre à son oreille des sons inaccoutumés ; parmi ceux qu'il reconnaît, ils lui prescrivent d'appeler consonne ce qui chez nous est appelé voyelle : enfin toute la doctrine grammaticale est exposée dans un langage qui, ne lui étant point encore connu, laisse tout obscur à sa pensée (VIII, 94-95).

8 N'étant ni arabophone ni arabisant, nous lirons la « grammaire » d'Erpenius en tant que « novice » européen désirant s'initier à l'arabe. N'était-elle pas d'ailleurs destinée à ce type de public ? Il s'agit, en termes modernes, d'un manuel d'arabe (littéral) langue étrangère pour des débutants déjà habiles en latin, et donc déjà " grammaticalisés » selon la tradition grammaticale gréco-latine, manuel qui est plus ou moins conforme aux manuels du temps qui commençaient par les " règles » d'une langue, morte ou vivante, avant de les utiliser dans « l'explication » de divers textes. Or chez Erpenius, ces « règles » sont, autant que nous puissions en juger (à l'aide de Grand'Henry 2000), une sorte de «transposition didactique » de la tradition grammaticale arabe, qui n'est adaptée que marginalement à la tradition gréco-latine. Il procède plus ou moins à la manière d'un J. Reuchlin cherchant, au début du XVIe siècle, à inculquer les " rudiments " de l'hébreu (biblique) à de futurs exégètes (Kessler Mesguish 2000 : 201-203) : «Reuchlin s'inspire de la tradition hébraïque avec ses trois parties du discours : nom, verbe, particule, [....] mais il 'récupère' malgré tout les catégories latines : le nom englobe pronom et participe, la particule recouvre l'adverbe, la conjonction, la préposition et l'interjection ».

9 Le Recueil des questions qui, contrairement à la grammaire d'Erpenius, fut sans doutevraiment « remis entre les mains » de Boisgirais par de Hauterayes, a aussi joué un rôle important dans sa préparation intellectuelle. Pour Michaelis (voir Hassler 2001 : 19-20), le « voyageur » - par quoi il entend non ce que l'on allait bientôt appeler un « touriste », mais un lettré préoccupé d'exégèse biblique - qui part pour l'Orient a besoin (Michaelis $1763^{18}$ )« de deux sortes de secours »: a) « la connoissance préalable de la langue du Pais que l'on veut visiter », par quoi il entend " une connoissance [...] puisée dans les principes de la Grammaire ", parce que cette connaissance aide "à avancer plus promptement dans la langue parlée des Arabes, lorsqu'il [le voyageur] vivra parmi 
eux »; b) qu'il sache à l'avance ce qu'il doit observer, d'où la nécessité de le " diriger ", et par " des questions précises lui déterminer les sujets sur lesquels on souhaiterait d'être éclairci ». Par exemple, remarquer « en quoi le langage de la vie commune s'éloigne des régies grammaticales connues de nos savans ", ou être attentif à " la maniere de châtrer ", parce que "l'Arabie est de tous les pais celui qui peut fournir les meilleurs éclaircissements sur les Loix de Moyse ". Il s'agit donc d'un guide pour savants chargés de recueillir, sur le terrain, des connaissances qui « se rapportent toutes ou peu s'en faut, à l'éclaircissement de l'Ecriture sainte ».

Ce que le Voyage doit à ce Recueil des Questions est manifeste, non seulement dans son mode d'exposition qui tient plus du rapport que du récit ${ }^{19}$, mais aussi dans nombre de ses réflexions. Ainsi, son premier chapitre inclut un long passage, qualifié par Gaulmier (1980 : 89) de " digression», qui ne fait que développer ce que Michaelis disait dans sa préface : « l'ignorance où sont la plupart des voyageurs de la langue arabe, et surtout de sa prononciation ", a pour conséquence " que leur oreille, novice à des sons étrangers, en a fait une comparaison vicieuse aux sons de leur propre langue ", d'où une " orthographe des noms propres » très disparate faisant que ce qui est écrit « en français chaik ou chêk, est écrit tour à tour schek, shekh, schech, sciek, selon qu'on l'a tiré de l'anglais, de l'allemand ou de l'italien » (II, 69-73). Et Volney de proposer, pour pallier ce " désordre d'orthographe qui confond tout ", d'assigner aux sons de l'arabe les " représentants » européens qui sont « les plus rapprochés des nôtres, en leur ajoutant des signes convenus ", ce qui serait " un premier pas vers une opération qui devient de jour en jour plus pressante et plus facile, un alphabet général qui puisse convenir à toutes les langues, ou du moins à celles de l'Europe » (II, 69-73). Première esquisse de sa "méthode alfabétique ${ }^{20}$ ». Ajoutons que les Questions de statistique, à l'usage des voyageurs (VII, 377-396) que Volney publiera en 1793 sont une laïcisation - à des fins non plus exégétiques, mais commerciales et militaires - des «questions » de Michaelis.

11 Mais le Voyage témoigne aussi de l'expérience que fit Boisgirais, en Egypte et en Syrie, d'un multilinguisme quotidien auquel il n'avait été jusqu'alors que peu accoutumé. Ainsi, toujours dans son Alfabet européen, rappelle-t-il que " dans le cours de (s)on voyage, (il) eu(t) l'occasion d'entendre parler dix à douze langues diverses » (VIII, 98), ajoutant en note :

Au Kaire, j'entendais l'arabe de la bouche du peuple, et le turc de la bouche des militaires et des effendis. Mon maître d'écriture était turc de Constantinople : j'eus l'occasion d'entendre les Gellâb, ou marchands d'esclaves noirs, parler éthiopien, et trois Malabars parler leur dialecte indou; dans Alep, outre l'arabe et le turc, j'entendais journellement l'arménien, le grec, plusieurs fois le kurde et le persan, sans compter l'allemand, l'anglais, le hollandais, le slavon, l'espagnol et l'italien, dans les maisons des Francs. En cette ville, il n'est pas rare de voir une seule maison se composer d'individus parlant cinq ou six langues, et les enfans les entendre sans les confondre » (ibid., note 1).

12 A quoi, il faut ajouter cette langue copte, langue plus liturgique que quotidienne, à laquelle Volney consacre, dans son Voyage, une remarque pour ainsi dire " précomparatiste » :

beaucoup de mots [du copte] sont comme des débris et les restes de l'ancien égyptien. Ces mots, examinés avec critique, ont une analogie sensible avec les idiomes des anciens peuples adjacents, tels les Arabes, les Ethiopiens, les Syriens et même les riverains de l'Euphrate; et l'on peut établir comme un fait certain que toutes ces langues ne furent que des dialectes dérivés d'un fonds commun (II, 69). 
13 Expérience d'un multilinguisme quotidien et réflexion sur la « filiation » des langues qui contrastent singulièrement avec ce que Volney appelle, dans le passage du Voyage relatif au monastère grec-catholique de Mar-Hanna, « le scientifique de l'arabe » :

Dans les premières années de ce siècle, les jésuites, profitant de la considération que leur donnait la protection de la France, déployaient dans leur maison d'Alep le zèle d'instruction qu'ils ont porté partout. [...] Les Latins d'Alep, excités par les jésuites, ne tardèrent pas de recommencer, comme autrefois, à argumenter contre les Grecs ; mais comme la logique exige une connaissance méthodique de la langue, et que les chrétiens, exclus des écoles musulmanes, ne savaient que l'arabe vulgaire, ils ne pouvaient satisfaire par l'écrit leur goût de controverse. Pour y parvenir, les Latins résolurent de s'initier dans le scientifique de l'arabe. L'orgueil des docteurs musulmans répugnait à en ouvrir les sources à des infidèles; mais leur avarice fut encore plus forte que leurs scrupules; et moyennant quelques bourses, la science si vantée de la grammaire et du nahou fut introduite chez les chrétiens " (italiques originales, III, 78).

14 Il revient plus longuement sur ce "scientifique de l'arabe » dans le chapitre intitulé « Des arts, des sciences et de l'ignorance »:

La seule science qui leur soit propre [aux Arabes], la seule qu'ils cultivent encore est celle de leur langue : et par étude de la langue, il ne faut pas entendre cet esprit philosophique qui, dans les mots, cherche l'histoire des idées pour perfectionner l'art de les peindre. Chez les Musulmans l'étude de l'arabe n'a pour objet que ses rapports à la religion : ils sont étroits, attendu que le Qôran est la parole immédiate de Dieu : or, comme cette parole conserve l'identité de sa nature, qu'autant qu'on la prononce comme Dieu et son prophète, c'est une affaire capitale d'apprendre nonseulement la valeur des mots employés, mais encore les accents, les inflexions, les pauses, les soupirs, les tenues, enfin tous les détails les plus minutieux de la prosodie et de la lecture. Il faut avoir entendu leur déclamation dans les mosquées, pour se faire une idée de sa complication. Quant aux principes de la langue, ceux de la grammaire seulement occupent pendant plusieurs années. Vient ensuite le Nahou, partie de la grammaire que l'on peut définir une science des terminaisons étrangères à l'arabe vulgaire, lesquelles se surajoutent aux mots, et varient selon les nombres, les cas, les genres et les personnes. Lorsqu'on sait cela, l'on est déjà compté parmi les savants. Il faut ensuite étudier l'éloquence ; et cela veut des années, parce que les maîtres, mystérieux comme des brames [sic : brahmanes], ne découvrent que peu à peu le secret de leur art. Enfin, l'on arrive aux études de la loi et au Faqah, ou science par excellence, qui est la théologie. Or, si l'on observe que la base perpétuelle des ces études est le Qôran; que l'on doit méditer à fond ses sens mystiques et allégoriques, lire tous les commentaires, toutes les paraphrases de son texte (et il y en a deux cents volumes sur le premier verset); [...]; l'on conviendra que l'on peut passer la vie entière à beaucoup apprendre et à ne rien savoir (III, 274-276).

Il y a là une sorte d'alacrité irrévérencieuse qui n'est pas sans lien avec les convictions " philosophiques » de Volney, lesquelles se heurtent au traditionalisme des religions tant chrétienne ${ }^{21}$ que musulmane ${ }^{22}$. D'où une conclusion proprement lapidaire : «Ce qui se passe en Asie depuis douze cents ans, peut en faire la preuve ; car si l'on voulait passer d'un sujet particulier à des considérations générales, il serait aisé de démontrer que les troubles des états, et l'ignorance des peuples dans cette partie du monde, sont des effets plus ou moins immédiats du Qôran et de sa morale " (III, 238). Et son dernier chapitre des Ruines proclamera bientôt qu'il " faut ôter tout effet civil aux opinions théologiques et religieuses " (souligné dans le texte : I, 242). Ce n'est donc pas par hasard si Boisgirais adopte, alors qu'il rédige son Voyage, le nom de Volney : il est devenu « voltairien ${ }^{23}$.» 


\section{Une Méthode nouvelle et facile d'apprendre les langues arabe, persane et turke, avec des caractères européens}

Ainsi sous-titrée, la Simplification comprend un Discours préliminaire et une Grammaire de la langue arabe qui est plus ou moins étendue au persan et au turc (alors écrit en caractères arabes).

Le Discours précise bien l'objectif visé et la méthode adoptée : Volney veut «faciliter les langues orientales ; [...] les rendre accessibles, presque populaires, en les ramenant à la condition des langues d'Europe dont elles ne diffèrent point essentiellement » (VIII, 189). Quand un Européen cherche à les apprendre, il se heurterait à deux difficultés, leur « prononciation » et leur " mécanisme ou construction » (ibid.). Volney admet que l'arabe offre à « nos oreilles des prononciations dont la nouveauté les étonne » (ibid.), mais pour le persan et le turc, la difficulté serait « presque nulle, leur prononciation étant presque aussi coulante et plus harmonieuse que celle d'aucune langue d'Europe » (VIII, 190). La difficulté de l'arabe serait, en outre, accentuée par la diversité de ses prononciations :

Il ne faut pas croire que l'arabe soit identique [nous soulignons] comme le français : au contraire, il subit des différences assez sensibles d'un canton à l'autre. Un Arabe d'Alger a de la peine à se faire entendre au Kaire ; un Arabe de Syrie comprend difficilement un Arabe d'Yemen : [...] d'où il est résulté une distinction d'arabe vulgaire et d'arabe littéral, par laquelle chaque canton appelle vulgaire ce qu'il usite, et littéral ce qui lui est étranger, parce que cet arabe étranger se trouve consigné dans des livres qui néanmoins ont cours dans toute l'Arabie (VIII, 203).

Et Volney de suggérer que l'arabe pourrait devenir tout aussi « identique » que le français, pour peu qu'émerge « parmi les Arabes un peuple dominateur et poli » (VIII, 203) qui soit à même de le " policer», à la manière sans doute dont le français le fut à partir du XVIIe siècle. Quant à la « construction » «aucune langue d'Europe » n'aurait (nous soulignons) « la régularité, ni la simplicité de l'arabe, encore moins du persan ; dans aucune, les phrases ne sont plus claires, plus méthodiques : c'est notre construction française "; seul le turc dérogerait « à cette clarté ", mais pas plus que l'allemand ou que le latin (VIII, 190). Autant de termes qui renvoient à la distinction proposée par Gabriel Girard en 1747, entre langues « analogues», parce que supposées conformes à la marche même de l'esprit, et langues «transpositives», qui ne jouiraient pas de cette conformité.

De ces similarités, il résulte que la difficulté principale de ces langues pour un Européen consiste « uniquement dans la figure des lettres, et dans le système vicieux de l'alfabet $»$ (VIII, 191).

Nos langues s'écrivent, plus (l'espagnol) ou moins (l'anglais, le français), comme elles se prononcent, alors que « dans l'arabe et dans ses analogues », seules « les consonnes, qui en sont la base principale, et les quatre voyelles longues » sont transcrites, les « trois voyelles brèves, qui jouent le plus grand rôle dans la prononciation, et qui en sont partie intégrante " étant "supprimées et sous-entendues » (VIII, 193). Il s'ensuit que leur lecture est « une divination perpétuelle, au point qu'il n'est aucun érudit arabe, persan ou turk, capable de lire couramment un livre s'il n'en a fait une préparation préalable » (VIII, 194). 
D'où la nécessité (nous soulignons) d' "abroger ce système, et lui en substituer un plus simple et plus parfait : or comme le système alfabétique d'Europe réunit une partie de ces conditions, comme il nous est déjà connu, familier, et que l'on peut l'étendre et le perfectionner, c'est faire tout d'un coup un pas considérable dans la connaissance des langues asiatiques, que de leur appliquer, et de peindre leurs prononciations par nos caractères » (VIII, 195).

2 Cette rencontre du " plus simple et plus parfait » avec ce qui "nous est déjà connu, familier " se retrouve souvent chez les Lumières. Non seulement chez l'abbé Girard (voir ci-dessus), mais aussi chez Dumarsais présentant à des élèves qui parlent le français un latin rangé didactiquement "selon l'ordre de la construction simple ", ordre présupposé « naturel » et conforme à « l'ordre primitif des pensées », mais qui se trouve être, par une coöncidence heureuse mais suspecte, celui même du français (Besse 1996). Il y a parfois ainsi chez les Lumières une propension à ériger en « nature » ce qui n'est que l'effet de leur propre « culture » (Besse 1998).

Le Discours se termine par une remarque révélatrice de la fascination qu'exerce le monde oriental sur Volney et de sa conviction qu'il faut - néanmoins ou en conséquence ? - le « civiliser » selon le modèle européen (nous soulignons) :

Et quand on observe l'heureuse organisation de ces peuples, comparée à leur arrièrement en civilisation et en connaissance, l'on est tenté de croire que la cause première de cet arrièrement n'a résidé que dans le vice de leur système d'écriture, qui, comme chez les Chinois, rendant l'instruction difficile, a, par une série de conséquences, rendu plus rare l'instruction, empêché la création des livres, leur publication, leur impression, et consolidé le despotisme des gouvernemens par l'ignorance des gouvernés (VIII, 202).

Nous lirons la Grammaire de la langue arabe de Volney à la façon dont nous avons lu celle d'Erpenius, en " novice » européen quelque peu informé des grammairiens du XVIIIe siècle. Elle est divisée en huit chapitres regroupables en quatre volets : a) « De la Langue Arabe, de ses Prononciations et de ses lettres alfabétiques » (chap. $1: 31$ pages, plus deux tableaux hors-texte, dont un in-folio) ; b) « Du Nom » (chap. II : 13 pages) ; c) « Des Pronoms personnels et possessifs, des Conjonctions et des Particules » (chap. III : également 13 pages) ; d) « Des verbes » (du chap IV portant ce titre au chap. VIII qui traite des "Verbes imparfaits et doublement irréguliers", soit une quarantaine de pages). Aucun chapitre n'est réservé à la syntaxe. L'ordre habituel des parties du discours s'y trouve certes un peu bousculé, mais les grammaires françaises du temps commençaient souvent ${ }^{24}$ par traiter des sons et des lettres, avant de passer aux parties du discours (réduites à trois classes par certains ${ }^{25}$ ) et parfois, mais pas toujours, à la syntaxe. A première vue, la grammaire arabe s'y trouve donc bien ramenée, selon le projet de Volney, au « déjà connu, familier » d'un Européen lettré.

Volonté qui se retrouve dans le premier chapitre : "la langue arabe, ainsi que nos langues d'Europe, est composée de trois élémens de prononciation qui sont $: \mathrm{I}^{\circ}$ Les voyelles, $2^{\circ}$ Les consonnes, $3^{\circ}$ Les aspirations ${ }^{26} "$ (VIII, 205), la voyelle par exemple, étant définie, curieusement, comme « tout son simple, indivisible, proféré par le gosier, sans le mélange d'aucun autre son qui en change la modification à l'oreille (sic) » (VIII, 205). A quoi Volney ajoute que les Arabes « ont encore avec nous cela de commun, d'avoir représenté chacun de ces élémens de la parole par des signes attachés à chacun d'eux, et appelés vulgairement lettres alfabétiques » (VIII, 207). Ce n'est donc que dans leur écriture que se manifestent " plusieurs différences » (ibid), qu'il « s'agit de faire disparaître » (VIII, 208). 
Sur les « vingt-huit lettres arabes », Volney considère que « dix-sept représentent des prononciations absolument les mêmes que dans notre langue française ", il les représente donc «par nos propres lettres » (ibid.). Les onze restantes peignent « des prononciations qui nous sont étrangères ", et il les représente donc par " des signes propres et particuliers » (VIII, 209), qu'il emprunte à l'alphabet grec ou qu'il invente. S'en prenant à " quelques grammairiens européens ", pour qui « les vingt-huit lettres de l'alfabet arabe seraient toutes des consonnes " (VIII, 208), il affirme que quatre d'entre elles « sont de véritables voyelles, savoir : $a$, i, ou et a guttural ou aïn » (VIII, 212), et fait observer que les Arabes « emploient dans leur écriture d'autres signes qui, pour n'être pas compris dans l'alfabet, n'en sont pas moins des caractères alfabétiques, de véritables lettres voyelles ", tels les "trois signes appelés motions ou points figurés " (ibid.). Il s'ensuit que l'arabe compte, aux yeux de Volney, « au moins sept voyelles trèscaractérisées ", celles transcrites par les " quatre lettres alfabétiques ou majeures » et celles qui le sont par les "points-voyelles " ( $a, i, o$ brefs). A quoi il s'ajoute que ces derniers, " appliqués aux voyelles majeures de l'alfabet, les modifient encore de manière qu'il en résulte de nouvelles voyelles, distinctes des unes et des autres, et formées à la manière de nos diphtongues ": ainsi l'alef suscrit par un de ces pointvoyelles "vaut â », mais souscrit par le même se prononce " exactement é " (VIII, 215-217). Volney aboutit ainsi à « effectivement douze » voyelles, auxquelles il adjoint « les trois nunnations ou nasalemens», qui sont des terminaisons " fréquentes des mots dans l'arabe littéral »(ibid.). D'où " un canon alfabétique bien plus étendu qu'on a voulu le croire jusqu'à nos jours ", en ce qu'il est composé de trente-six sons différents (12 voyelles +2 aspirations +22 consonnes), auxquels sont adjointes, hors classement, les trois nunnations. C'est ce « canon » que présente, en six colonnes, le tableau hors-texte in-folio intitulé : " Alfabet arabe, transposé en caractères européens, à l'usage des voyageurs et négocians en Asie et en Afrique ».

Dans les chapitres suivants, Volney cherche de même à « ramener » la morpho-syntaxe de l'arabe à celles des langues européennes, mais il est loin d'y parvenir aussi aisément que pour les « sons ». Le début du second chapitre ("Du Nom ») est de ce point de vue éclairant. D'une part, l'arabe est supposé semblable aux langues de l'Europe : « En arabe comme en français, le discours est composé de trois parties principales, $1^{\circ}$ le nom (de l'agent), $2^{\circ}$ celui de l'action ou le verbe, et $3^{\circ}$ les particules qui lient l'agent à l'action, c'est-à-dire le nom au verbe » (VIII, 232), tripartition alors relativement admise ; « rien n'est plus simple que la déclinaison du nom en arabe vulgaire; il est le même à tous les cas qui ne se distinguent que par les particules, ou par le sens de la phrase » (VIII, 232), déclinaison voisine donc, là aussi, de celle du français telle que la présentaient de nombreux grammairiens depuis le $\mathrm{XVI}^{\mathrm{e}}$ siècle ; et si cette " équivoque des cas » ne se retrouve pas en " arabe littéral ", c'est que cette variété fonctionne " comme " le grec et le latin (VIII, 234). D'autre part, dès que Volney se veut plus précis, il se trouve contraint, ou bien d'avoir recours au "scientifique de l'arabe " (" 1'l dans al se perd devant treize consonnes, appelées solaires ", VIII, 233), ou bien de s'en remettre à la simple pratique (" c'est à l'usage d'enseigner cela, et non à l'écriture de le tracer ", parce que "les signes imaginés par les grammairiens pour diriger cette manière d'écrire, sont aussi ridicules que si chez nous l'on écrivait [...] : il zon t'écri t'a Rome " (VIII, 234). Difficulté qui est encore plus évidente dans les chapitres suivants. Le troisième, qui porte sur les adverbes, prépositions, conjonctions et autres « particules formées de mots invariables " (VIII, 256), se borne à les énumérer dans des listes bilingues : «Quand, lorsque : matâ, lamma » (VIII, 257). Et les six chapitres consacrés au 
verbe ont constamment recours au "scientifique de l'arabe», sans doute parce que " c'est dans le verbe en général que la langue arabe développe davantage [...] sa différence avec les langues d'Europe » (VIII, 260).

Enfin, au lieu d'appliquer sa Grammaire à une sourate du Coran comme le faisait Erpenius, Volney l'applique à « quelques proverbes arabes, qui achèveront de donner une idée de (s)a méthode de transposition » (VIII, 301). Notons que si les neuf premiers sont «transposés » selon l'arabe littéral, les suivants le sont selon l'arabe vulgaire, « sans finales grammaticales » (VIII, 307).

\section{Quelques « leçons » tirées d'un relatif échec}

Gaulmier (1945-1946 : 116) juge que la Grammaire « est évidemment très rudimentaire et ne saurait être comparée au chef d'œuvre d'érudition qu'est celle de Silvestre de Sacy ", mais il ajoute que, destinée " aux non savants ", elle " offre l'incontestable avantage de la simplicité ", celle "d'un compromis entre l'arabe littéral et l'arabe parlé, ne retenant que le dénominateur commun nécessaire pour lire un texte facile et, en même temps, pour comprendre une conversation sans pédantisme ". Jugement qu'il reprend, à quelques nuances près, dans sa thèse (1980 : 318-319). Notre connaissance de l'arabe est insuffisante pour que nous puissions le contester sur ce point, même si Gaulmier accompagne son jugement de commentaires éminemment critiquables ${ }^{27}$. Il nous semble mieux avisé quand il précise - dans une note qui semble un repentir $(1980: 318$, note 3) - que « la transcription de Volney ne prétend nullement être une transcription phonétique capable de figurer tous les détails mouvants d'un dialecte », qu'il « faut y voir plutôt une translitération logique ", même s'il nous semble mal percevoir les implications didactiques que peut avoir cette translittération que nous préférons dire raisonnée.

Constatons d'abord que la "méthode alfabétique " n'eut pas le succès qu'en espérait Volney. Dans son « Epître dédicatoire à l'Honorable Société Asiatique, séante à Calcutta " qui sert d'introduction à l'Alfabet européen, il rappelle que, malgré l'opposition des " arabistes de Paris ", son "système de transcription européenne fut admis par une majorité des deux tiers » d'une commission - où il avait lui-même proposé que siègent " trois savans éminens par leur connaissance mathématiques ${ }^{28}$ » - qui fut chargée, en 1803, de décider de la meilleure " double nomenclature arabe et française » pour « le somptueux ouvrage de la Description de l'Egypte » (VIII, pp. IX-XI). Il y revient dans son Epitre à Messieurs les membres de l'Académie française qui ouvre son Hébreu simplifié : « Ma méthode fut d'abord nettement repoussée, il y a vingt cinq ans; ensuite on l'a regardée comme fondée en principe ; on l'a mise en pratique ${ }^{29}$. Les anciens tiennent bon et n'en veulent point ; les jeunes gens l'examinent et la discutent : avec le temps, vous verrez qu'elle fera secte " (VIII, 325). Et de nouveau dans son Discours sur l'étude philosophique des langues : "Les principes sur lesquels mon système est fondé sont aujourd'hui reconnus pour aussi solides, aussi clairs que ceux de l'algèbre ${ }^{30}$; mais leur application [...] sont, et seront combattus par les anciennes habitudes » (I, 408). Il existe, à nos yeux, d'autres raisons que la routine au relatif échec de sa "méthode alfabétique ». Une double contradiction nous semble en miner l'efficacité pratique.

D'une part, « l'alfabet européen », du moins tel qu'il est proposé dans la Simplification, est effectivement plus une translittération de l'alphabet arabe qu'une écriture fondée sur une phonétique ou une phonologie de la langue arabe, fût-elle réduite à son " 
littéral ». Volney en conclut le premier chapitre par ces mots symptomatiques (nous les soulignons) : "Tous les signes de l'écriture arabe s'y trouvent rassemblés ; tous y reçoivent un équivalent en caractères européens déjà existans ou de convention; ils sont tellement combinés qu'ils forment un système d'écriture homogène et régulier avec lequel l'arabe, le persan, le turk, et toutes les langues peuvent s'écrire comme on les parle» (VIII, 218). Mais, pour ce qui est de l'arabe, il aurait été plus exact de dire : « comme on l'oralise quand on apprend à lire son écriture traditionnelle auprès de maitres qualifiés ". Car la fonction de cette écriture est d'abord de transcrire et de maintenir la variété « littérale » de l'arabe qui, au temps de Volney comme au nôtre, n'est plus - à supposer qu'elle le fût au VII ${ }^{e}$ siècle dans une partie de la péninsule arabique - acquise nativement, variété que Sacy put donc décrire grammaticalement sans être à même de parler arabe. Volney avait pourtant bien observé, dans son Voyage (voir ci-dessus, dans notre première partie, la citation extraite du chapitre « Des arts, des sciences et de l'ignorance»), la pédagogie multiséculaire de cette variété de référence pour tout arabophone, mais il pensait alors que la "science » européenne était à même de se passer d'un " art » si apparemment opposé à ses critères ${ }^{31}$. Et l'on a quelque peine à comprendre comment cette translittérarisation pourrait être utile à " des voyageurs et négocians " qui, à l'instar de Volney, ne sont guère soucieux de " littérature orientale ${ }^{32}$ ", même si l'on veut bien admettre que la méthode de Volney a, " entre autres mérites, de rendre nuls cinq signes usités dans l'arabe ${ }^{33} »$ et de supprimer "plus d'un quart de la grammaire d'Erpenius" (VIII, 229-230). Ajoutons que cette translittérarisation prive le "novice » européen d'une grande partie de la cohérence sémiotique interne à l'écriture arabe : le tableau in-folio qui la résume ne facilite guère, en listant les uns au-dessous des autres les caractères de l'alphabet arabe, le repérage des formes voisines graphiquement, alors que l'« Alfabet arabe selon l'ordre vulgaire " a le mérite de les rapprocher. Si l'arabe "savant ou littéral " perdure, y compris oralement, par le truchement de celui-ci, comment pourra-t-on s'en passer durablement ? Et dès lors, pourquoi se donner la peine d'apprendre "l'alphabet européen » de Volney ${ }^{34}$ ?

D'autre part, 1' " alphabet arabe selon l'ordre vulgaire » est difficilement dissociable de ce «scientifique de l'arabe » que Volney brocarde, dans son Voyage, mais dont il ne parvient pas, dans sa Grammaire, à totalement se passer. Le "voile hiéroglyphique " (VIII, 194) qui, à ses yeux, couvre l'écriture arabe peut certes être en partie levé par sa trans-littération - dont il faut cependant comprendre et mémoriser les conventions -, mais il reste à apprendre ce qui est propre au « littéral » de cette langue étrangère, qui est difficilement transmissible au moyen de catégories et raisonnements grammaticaux - fussent-ils réputés généraux voire universels - qui relèvent d'une tradition autre que celle qui norme ce « littéral » depuis des siècles.

Si la " méthode alfabétique», telle que Volney l'exposait en 1795, nous paraît peu défendable d'un point de vue didactique ${ }^{35}$, elle a joué un rôle important dans l'évolution de sa pensée linguistique, telle qu'il la formule, plus de vingt ans après la Simplification, dans ses trois derniers ouvrages, quand il cherche à la généraliser à d'autres langues que l'arabe, le persan et le turc. Bornons-nous, en conclusion, à quelques passages, qui nesont certes pas radicalement originaux pour son temps, mais qui nous semblent attester d'une réelle sensibilité à ce que l'on commence à appeler la « linguistique ».

L'Alfabet européen est certes fondé sur la même conviction que la Simplification, à savoir que « si l'organisation humaine est la même en Asie qu'en Europe, le langage dans ce 
pays-là doit être composé d'élémens semblables aux nôtres » (VIII, 95). Mais Volney y définit ces " élémens " différemment, s'y montrant attentif à la valeur " distinctive " des sons, tout comme P. Passy et D. Jones le seront ${ }^{36}$, à la fin du XIXe siècle, dans leur l'Alphabet Phonétique International (API). Pour nous en tenir aux voyelles, dont le nombre serait de " quinze ou vingt», il clarifie nettement (nous soulignons) la définition qu'il en donnait dans la Simplification (« qui en change la modification à l'oreille ») : " l'analyse de quatre ou cinq alfabets seulement de langues vivantes, en fournit presque ce dernier nombre, tellement distinctes qu'on ne peut les substituer ou les confondre, sans changer les sens des mots " (VIII, 6); ou bien " la différence que nous signalons entre à clair et â profond est tellement réelle, que si l'on prononce l'un au lieu de l'autre, le sens des mots en certains cas en sera changé» (VIII, 29). Après avoir classé les sons d'après la " nature des organes » engagés par leur articulation, Volney relativise ce classement, entre autres par cette remarque d'ordre nettement acoustique, qui préfigure la notion de « philtre phonologique » (Troubetskoy 1949 : 54-55):

Cette manière a l'inconvénient de ne point marquer à l'oreille du disciple, surtout étranger, la différence entre une consonne qui lui est connue et sa pareille qui ne lui est pas : je prends, par exemple, un Arabe, qui, dans sa langue, n'a que le Bé, et point le Pé : si je lui dis que Bé n'est point Pé, il ne me comprend point, il répète Bé; mais si je lui dis que Bé n'est point $P o$, son oreille est avertie de la différence, et son esprit commence à la chercher (VIII, 62).

En 1795, Volney écrivait qu'il ne fallait « pas croire que l'arabe soit identique comme le français »; en décembre 1819, il s'adresse ainsi aux membres de l'Académie française :

chez les nations même civilisées, [...] les classes et professions où le temps de l'homme et de la famille est absorbé par les soins de la subsistance [...] ne connaissent de la langue nationale que la portion qui leur est nécessaire : amenez un paysan, un ouvrier dans nos assemblées scientifiques, vous verrez combien de mots ils ne comprennent pas ; faites-les suivre un raisonnement ou une narration, vous verrez qu'ils n'ont pas l'usage de plusieurs modes et temps de nos verbes. On se fait des illusions, lorsqu'on parle des nations comme des corps sociaux homogènes à la manière des corps physiques; elles ne sont que des confédérations de peuples différents qui [...] ont des sphères d'idées, et par conséquent des dictionnaires de mots très-différents. (I, 414)

Manifestement le français n'est plus aussi « identique » qu'en 1795, et Volney est moins certain qu'au temps de son Catéchisme du citoyen français (1793) de la possibilité de déduire « de l'organisation de l'homme et de l'univers » les " principes physiques de la morale. » En 1795, l'arabe et le persan sont, tout comme le français, des langues « analogues »; le chapitre « De la syntaxe » de son Hébreu simplifié s'ouvre par cette mise en garde ironique :

En comparant diverses langues, les grammairiens se sont aperçus qu'il existait deux manières de construire la phrase [...] : ils ont appelé l'une construction directe ou naturelle, et l'autre construction par inversion.

Il n'est pas besoin de dire que par naturelle et directe on entend la nôtre ; car la manie humaine est que chacun tienne son habitude pour nature : à qui persuaderez-vous que nos antipodes soient aussi droits que nous? (VIII, 454).

Volney ne croit manifestement plus à la métaphysique de la Grammaire générale, et ce n'est pas par hasard si, dans son Discours sur l'étude philosophique des langues, il rappelle les travaux d'un grand nombre de grammairiens européens depuis le XVI ${ }^{e}$ siècle, mais ni ceux de Port-Royal ni ceux des grammairiens de l'Encyclopédie. Non qu'il dédaigne " approfondir des connaissances métaphysiques telles que la formation des idées et leur manifestation dans le langage » (I, 378), mais parce que si son étude " philosophique » 
des langues prend « l'observation des faits [...] comme préliminaire indispensable à toute théorie » (I, 401), c'est afin d'expliquer « comment elles naissent et se forment, comment elles s'accroissent, s'établissent, s'altèrent et périssent ", quelle sont « leurs affinités ou leur différences, leur filiation » (I, 375-376). Projet « encore neuf et difficile » (I, 376), qui sera bientôt celui de la grammaire comparée et historique. Le XIX ${ }^{\mathrm{e}}$ siècle a vu Volney en tant qu'historien ${ }^{37}$ ou philosophe pré-positiviste ${ }^{38}$; le $\mathrm{XX}^{\mathrm{e}}$ siècle s'est plutôt intéressé au voyageur (en Orient ${ }^{39}$ et aux Etats-Unis ${ }^{40}$ ), et à $l^{\prime}$ homme politique ${ }^{41}$; le XX ${ }^{e}$ siècle s'intéressera peut-être davantage au linguiste.

\section{BIBLIOGRAPHIE}

\section{Sources primaires}

BUFFIER, Claude (1731) : Grammaire françoise sur un plan nouveau. Nouvelle édition [...]. Par le P. Buffier, de la Compagnie de Jésus. A Paris, chez Marc Bordelet.

ERPENIUS (Thomas Van Erpe ou Van Arpen, dit) (1638) : THOMAE ERPENII, Rvdimenta Linguae Arabicae [1613]. Accedant eiusdem Praxis Grammatica ; \& Consilium de studio ARABICO féliciter instituendo. Lvtetiae Parisiorvm, Sump-tibus Societatis Typographicae. Librorum Officij Ecclesiastici, lussus regis constituae.

MICHAELIS, Johan David (1763) : Recueil de Questions proposées ci une société de savans qui, par ordre de sa Majesté Danoise, font le voyage d'Arabie. Par Monsieur Michaelis, Conseiller de Cour de S. M. Britannique, Professeur en Philosophie, et Directeur de la Société Royale de Göttingen. Traduit de l'Allemand. A Francfort sur le Mayn, chez Jean Gottlieb Garbe.

RADONVILLIERS, Claude-François Lizarde de (1768) : De la manière d'apprendre les langues. Paris, Saillant.

VOLNEY(1821) : Euvres complètes de Volney. Paris, Bossange frères, en huit volumes.

VOLNEY(1826) : CEuvres de C. F. Volney. Paris, Parmantier, Libraire, rue Dauphine ; Froment, Libraire, Quai des Augustins, en huit volumes.

VOLNEY(1827) : Oeuvres choisies de C.-F. Volney. Paris, Baudouin Frères Editeurs, Achille Désanges Librairie.

VOLNEY(1837) : Euvres complètes. Paris, Didot, en un volume.

VOLNEY(1989) : CEuvres I-II. Textes réunis par Anne et Henry Deneys. Paris, Fayard, « Corpus des Oeuvres de philosophie en langue française », en deux tomes.

\section{Sources critiques}

BESSE, Henri (1996) : « Beauzée et Radonvilliers ou la méthode de Du Marsais démembrée », Documents pour l'histoire du français langue étrangère ou seconde 17, 61-85. 
BESSE, Henri (1998) : « Nature et seconde nature dans l'enseignement/apprentissage des langues ", Revue canadienne de linguistique appliquée, vol. 1, n 1 -2, 29-51.

BUISSON, Ferdinand (1911) : article « Volney », Le Nouveau dictionnaire de Pédagogie. Paris, Librairie Hachette et Cie, 2047-2049.

GAULMIER, Jean (1945-1946) : « Volney et la pédagogie de l'arabe », Bulletin d'Etudes Orientales (Institut Français de Damas), tome XI, 113-120.

GAULMIER, Jean (1980) : L'idéologue Volney 1757-1820. Contribution à l'Histoire de l'Orientalisme en France. Genève-Paris, Slaktine Reprints. [Thèse d'Etat soutenue en juillet 1949, publiée à Beyrouth en 1951 par l'Imprimerie catholique.]

GRAND'HENRY, Jacques (2000) : Grammaire arabe à l'usage des Arabes. Traduction française et commentaires des Eléments d'arabe, morphologie et syntaxe, II, de Rachid Chartouni (Beyrouth). Louvain-la-Neuve, Peeters.

HASSLER, Gerda (2001) : « La discussion sur l'universalité de la langue française et la comparaison des langues : la rupture épistémologique », in KOK ESCALLE - MELKA réd. (2001), 15 - 39.

KESSLER MESGUISH, Sophie (2000) : « 4209. Reuchlin, Johanes [Capnion] », in Histoire, Epistémologie, Langage, « Corpus représentatif des grammaires et des traditions linguistiques » (tome 2), Hors-série n 3, 201-203.

KOK ESCALLE, Marie-Christine, MELKA, Francine (2001), Changements politiques et statut des langues. Histoire et épistémologie 1780 - 1945. Amsterdam - Atlanta, Rodopi.

Le Coran (1977). Traduction par D. Masson. Revue par Dr. Sobhi EI-Saleh. Essai d'interprétation du Coran inimitable. Beyrouth, Dar Al-Kitab Al-Lubnani.

MEYNET, Roland \& alii (1979) : Du Golfe à l'Océan. Paris, Didier.

TROUBETSKOY, N. S. (1949) : Principes de phonologie [1939]. Paris, Klincksieck.

\section{NOTES}

1. Ce n'est qu'à partir de la troisième édition, celle de l'An VII (1799), que le titre en deviendra le Voyage en Egypte et en Syrie..., titre plus conforme à la chronologie de son périple.

2. Publication qui n'inclut que les Ruines, les Leçons d'histoire et un Discours de Lucien sur la manière d'écrire l'histoire.

3. Daru cite en note : De la Simplification des langues orientales (1795), L'Alphabet européen (1819), et L'Hébreu simplifié (1820).

4. On sait que Silvestre de Sacy, auteur d'une grammaire de l'arabe dont les spécialistes actuels reconnaissent les mérites, ne parlait pas la langue qu'il décrit.

5. En particulier, les Euvres complètes (Volney 1837) rééditées jusqu'en 1900, qui ne reprennent ni la Simplification ni les Vues nouvelles ni l'Alfabet européen. Ou bien l'édition récente (Volnet 1989), qui republie les libelles révolutionnaires exclus des éditions « complètes » antérieures, mais qui ne reprend ni le Voyage ni la plupart des textes relatifs à la « méthode alfabétique ».

6. Cette édition s'ouvre une « Notice sur la vie et les écrits de C.-F. Volney » d'Adolphe Bossange.

7. Le premier tome reprend cette « Notice... », pp. I-XLIX.

8. Nous nous bornerons à en donner le tome en chiffres romains et la (les) page(s) en chiffres arabes.

9. Cette « Esquisse » n'est pas sans approximations et oublis, par exemple Adolphe Bossange y est prénommé Alphonse (p. XXXVII), et les Oeuvres choisies de C.-F. Volney de 1827 n'y figurent pas. 
Et il en va à peu près de même chez les éditeurs de 1989, qui s'appuient sur Gaulmier sans vérifier ses dires.

10. Veuve du fermier général Claude-Adrien Helvétius auteur d'un ouvrage célèbre (De l'esprit, 1758), elle tenait alors un salon à Auteuil.

11. Paul-Henri Thiry, baron d'Holbach, un des quelque 140 auteurs de l'Encyclopédie qui publia, en 1761, Le Christianisme dévoilé, dont Volney se souviendra dans les Ruines

12. Il finira par paraître dans le Journal des Savons en janvier 1782.

13. II s'agit de la « noble et trilingue académie » dont Guillaume Budé avait obtenu, au grand dépit de la Sorbonne, la création par François 1er en mars 1530. Dite Souvent « collège des trois langues » (grec ancien, arabe et hébreu), elle est devenue notre actuel Collège de France.

14. Cette note date de la troisième édition du Voyage (1799), Bonaparte étant parti pour l'Égypte en mai 1798. Venture de Paradis, qui mourut devant Saint-Jean d'Acre, était, selon Volney, «l'un de nos plus habiles drogmans en turk et en arabe », et lors d'une mission près du dey d'Alger, en 1787, il avait dressé " une Grammaire et un recueil de mots berbères très considérables ", dont Volney s'inspirera dans son Hébreu simplifié (VIII, pp. 483-485).

15. Thomas Van Erpe ou Van Arpen, dit Erpenius (15847-1624), professeur de langues orientales à l'université de Leyde. Sa Grammatica arabica fut d'abord publiée à Leyde en 1613 (Thomas Erpenii Grammatica arabica. Leidae : in officina Raphelengiana, 1613. in- $4^{\circ}$, VIII-124 p.), puis à Paris en 1620. Il a également publié une " grammaire chaldaïque», à laquelle fait allusion Volney dans son Hébreu simplifié (VIII, p. 373, note 1).

16. Gaulmier avance (1980: 33$)$ que (nous soulignons) « le principal mérite du bon Leroux des Hautesrayes fut de mettre entre les mains de Boisgirais la grammaire arabe d'Erpenius, dans l'édition de Michaelis [référencée en note : Johan David Michaelis : Erpenii arabische Grammatik abgekürzt volstandiger und leitchter gemateli. Gottingen, 1771], et de recommander ainsi à l'attention de son jeune étudiant un autre ouvrage du même Michaelis, le Recueil des questions proposées à une société de savants qui, par ordre de sa Majesté danoise, font le voyage d'Arabie, dont la traduction française venait de paraître à Amsterdam et à Utrecht en 1774 »(ibid.). Deux affirmations qui ne sont pas étayées plus avant et qui nous paraissent contestables.

17. Nous n'avons pas pu consulter l'édition de Michaelis mais, grâce à l'amabilité de G. Bohas, une édition de 1638 imprimée à Paris, dont l'épître dédicatoire et la préface (Thomae Erpenii Consilium de studio Arabico féliciter instituendo) sont datées du 22 mars 1620 (Erpenius 1638).

18. Ni la Préface ni l'Instruction signée « Frederic R. », c'est-à-dire le roi du Danemark, ne sont numérotées.

19. Le comte Daru dira que Volney y « évite avec soin de se mettre en scène » (Volney $1827: 7$ ) et Gaulmier (1980: 67) qu'il lui donne la forme « d'une dissertation très impersonnelle ».

20. Une note, datant de l'édition de 1799 , signale qu'il s'agit de l'ouvrage "intitulé la Simplification des langues orientales » (II, 73, note 2).

21. Une seule citation : "Les moines de Mar-Hanna qui ont des livres, et qui entretiennent des relations avec Rome, ne sont pas à cet égard moins ignorants que les autres. Jamais, avant mon séjour, ils n'avaient ouï dire que la terre tournât autour du soleil, et peu s'en fallut que cette opinion n'y causât scandale : car les zélés, trouvant que cela contrariait la sainte Bible, voulurent me traiter en hérétique [...] J'en fus quitte pour ne point prendre la rotation sur mon compte, et pour la restituer à nos savants, qui passent sûrement chez les moines pour des visionnaires » (III, 273-274).

22. En atteste le chapitre que Volney consacre à « l'influence de la religion » musulmane, où il récuse la double lecture que l'on fait encore de nos jours du Coran : celle qui en fait la source du droit musulman : «Quiconque lira le Qôran, sera forcé d'avouer qu'il ne présente aucune notion ni des devoirs des hommes en société, ni de la formation du corps politique, ni des principes de l'art de gouverner, rien en un mot de ce qui constitue un code législatif» (III, 235-236) ; et celle qui en fait une lecture plus spirituelle : «Le reste n'est qu'un tissu de phrases vides de sens ; une 
déclamation emphatique d'attributs de Dieu qui n'apprennent rien à personne ; une allégation de contes puérils, de fables ridicules [...]. Que si, à travers le désordre d'un délire perpétuel, il perce un esprit général, un sens résumé, c'est celui d'un fanatisme ardent et opiniâtre » (III, 236).

23. Rappelons que Voltaire a écrit une tragédie ("Le fanatisme, ou Mahomet le Prophète», représenté d'abord à Lille en 1741) qui atteste, en alexandrins, d'une lecture du Coran voisine de celle de Volney.

24. Celles de P. Restaut et de N. F. Wailly, qui en traitent en fin d'ouvrage, faisant plutôt figure d'exception.

25. Le Père $\mathrm{Cl}$. Buffier avait publié, en 1709 , une Grammaire française sur un plan nouveau, où il proposait (Buffier 1731 : 133) de s'en tenir à «trois parties du langage qui sont, comme j'ai dis [...], les noms, les verbes, les modificatifs», et une réduction analogue, mais différente quant à certaines "parties du discours», se retrouve dans Radonvilliers (1768:36) divisant " en trois classes tous les mots d'une Langue ».

26. Volney entend par là le « h français » de honte et le « h très-dur » de l'arabe « horriàt, liberté.

27. II avance, par exemple, que " l'effort linguistique " de Volney " s'inscrit dans le grand dessein de compréhension universelle » qui a suscité, en son temps, « la chimère d'un alphabet commun », citant à ce propos « la 'pasigraphie' de Maismieux » (1980:314), C'est oublier que, contrairement à la méthode de Volney, la « Pasigraphie n'enseigne aucune langue ».

28. Une note précise qu'il s'agit de «MM. Monge, Berthollet, La Croix ».

29. Une note précise : "Sur la carte de l'Egypte».

30. Allusion à l'un des arguments que «MM. Monge, Berthollet, La Croix » avaient avancés, en 1803, pour faire pencher la balance en sa faveur.

31. Rappelons que l'Eglise de France avait condamné la proposition de J.-J. Rousseau voulant qu'Emile n'apprît rien par coeur.

32. A l'objection qu'avec sa méthode, on ne pourra pas lire les ouvrages écrits en arabe, Volney rétorque dans sa Simplification: "que presque tous les livres arabes, persans et turks, vraiment utiles et curieux, sont traduits dans nos langues d'Europe : qu'il en reste peu qui méritent la peine d'apprendre leurs langues : que malgré l'enthousiasme de quelques amateurs de la littérature orientale, elle est infiniment au-dessous de l'opinion que l'on s'en fait » (VIII, 196-197). 33. II s'agit du « repos d'une consonne », du redoublement de certaines lettres, de la « hamza qui avertit qu'il y a un $\mathrm{n}$ absent », et de la madda ou « extenseur de l'alef final ».

34. Un problème voisin se retrouvera quand, au temps de la "méthode directe ", on essaiera d'enseigner la prononciation des langues étrangères au moyen de l'Alphabet Phonétique International.

35. Encore qu'elle pourrait l'être. Un manuel d'arabe relativement récent (Meynet \& alii 1979) développe un projet assez voisin : enseigner à des débutants, mais en n'usant d'abord que de l'oral, un arabe « moyen » ou « intermédiaire » entre l'arabe « littéral » et les arabes parlés du Moyen-Orient.

36. A notre connaissance, ni l'un ni l'autre ne se réfèrent à Volney.

37. Voir ses oeuvres « choisies » ou « complètes » publiées après 1826.

38. F. Buisson (1911, 2047-2049) s'en tient aux Ruines et au Catéchisme.

39. Le Voyage a été réédité par Gaulmier en 1959.

40. Volney est l'auteur d'un Tableau du climat et du sol des Etats-Unis d'Amérique, paru d'abord en 1803.

41. La republication, en 1989 , de ses pamphlets et libelles révolutionnaires (1788-1795) en atteste. 


\section{RÉSUMÉS}

C.-F. Volney a passé plus de vingt-cinq ans de sa vie au « projet d'appliquer à l'étude des idiomes de l'Asie une partie des notions grammaticales acquises sur les langues européennes. » Projet lié à sa tentative d'apprentissage de l'arabe avant son départ pour l'Orient et à l'expérience qu'il y fit d'un multilinguisme quotidien. L'échec relatif que connut ce projet le conduisit, à la fin de sa vie, à remettre en cause une partie des présupposés de la Grammaire générale, base de sa tentative de réduction des langues orientales à la « science » européenne.

C.-F. Volney spent more than 25 years of his life in « a project which dealt with grammatical ideas acquired with European languages applied to the study of the languages of Asia. " This project was linked to his own attempt to learn Arabic before his departure for the Orient, and to the daily use of several languages he witnessed when he got there. The relative lack of success of this project led him, towards the end of his life, to question some of the assumptions of General Grammar, which he had used as a basis in his attempt to align Asian languages and European " science. »

\section{INDEX}

Keywords : Volney, language teaching, alphabetic method, Arabic, Hebrew, oriental languages Mots-clés : Volney, enseignement des langues, méthode alphabétique, arabe, hébreu, langues orientales

\section{AUTEUR}

\section{HENRI BESSE}

Ecole normale supérieure Lettres \& Sciences humaines 